\title{
Gesamterneuerungswahlen 2020 der FMH: Aufruf für Kandidierende
}

\section{Jürg Schlup}

Dr. med., Präsident der FMH

\section{Im Rahmen der Ärztekammer vom 28./29. Oktober 2020 finden die Gesamterneue-} rungswahlen aller Organe der FMH für eine neue Legislatur statt.

Gewählt werden:

a) die sieben Mitglieder des Zentralvorstandes der FMH inklusive Präsident und zwei Vizepräsidenten

b) der Präsident des SIWF

c) der Präsident und die vier Vizepräsidenten der Standeskommission

d) die fünf Mitglieder der Geschäftsprüfungskommission

e) die Mitglieder der Delegiertenversammlung (DV).

Kandidatinnen und Kandidaten für die oben aufgeführten Funktionen a) bis d) haben die Möglichkeit, sich in der Schweizerischen Ärztezeitung in strukturierten Porträts vorzustellen. Bitte beachten Sie, dass die von der Ärztekammer zu wählenden Personen gemäss Art. 22 der Statuten der FMH mit Erreichung ihres 68. Altersjahres aus dem Amt ausscheiden, sofern die Ärztekammer im Einzelfall keine Ausnahme beschliesst.

Ein Anforderungsprofil der Kandidatinnen und Kandidaten für den Zentralvorstand (ZV) sowie das Formular zur Publikation in der SÄZ sind bei den Präsidentinnen und Präsidenten der in der Ärztekammer vertretenen Organisationen oder bei deren Sekretären und Sekretariaten erhältlich. Neben den statutarischen Voraussetzungen (Facharzttitel und FMH-Mitgliedschaft) sind unter anderem Voraussetzungen wie politische und standespolitische Erfahrung, Führungskompetenz und Sprachkompetenzen gefordert.

Die Angaben der Kandidatinnen und Kandidaten benötigen wir bis spätestens am 24. Juli 2020 per E-Mail an wahlen2020[at]fmh.ch. Später eintreffende Angaben können nicht mehr in der Ärztezeitung publiziert werden. Ihre Fragen zu den Gesamterneuerungswahlen nehmen wir gerne per E-Mail an wahlen2020[at]fmh.ch entgegen.

Die bereits bekanntgegebenen Kandidaturen sind weiterhin gültig, werden im September nochmals in der Schweizerischen Ärztezeitung publiziert und müssen daher dem Generalsekretariat nicht noch einmal gemeldet werden. 